\title{
Review of the innovative H\&CD designs and the impact of their configurations on the performance of the EU DEMO fusion power plant reactor
}

\author{
T. Franke, P. Agostinetti, G. Aiello, K. Avramidis, Ch. Bachmann, A. Bruschi, G. Federici, \\ S. Garavaglia, G. Granucci, G. Grossetti, J. Jelonnek, J.-M. Noterdaeme, A. Simonin, T. Scherer, \\ P. Sonato, D. Strauss, M. Q. Tran, A. Valentine, P. Vincenzi, R. Wenninger, S. Zheng
}

\begin{abstract}
Heating \& Current Drive (H\&CD) systems are being investigated for a demonstration fusion power plant DEMO to deliver net electricity for the grid around 2050 [1],[2]. Compared to ITER, which has to show the generation of 500 MW thermal power, the target of DEMO is the successful production of 300 to $500 \mathrm{MW}$ electrical power to the grid and to aim for a self-sufficient Tritium fuel cycle [3]. Three H\&CD systems are under development for DEMO in Europe, the Electron Cyclotron (EC) System, the Neutral Beam Injection (NBI) System and the Ion Cyclotron (IC) System.

Based on present studies [4] for plasma ramp-up, rampdown and flat top phases, to be further validated in more detailed simulations, the assumed total launched power needed from the H\&CD system in DEMO is in the range of 50-100 MW, to be provided for plasma heating and control.

The paper describes the designs and $R \& D$ status of selected H\&CD systems considered for their deployment in the EU DEMO. It was always considered that different H\&CD
\end{abstract}

Manuscript submitted on $28^{\text {th }}$ June 2016. This work has been carried out within the framework of the EUROfusion Consortium and has received funding from the Euratom research and training programme 2014-2018 under grant agreement No 633053. The reviews and opinions expressed herein do not necessarily reflect those of the European Commission.

T. Franke and R. Wenninger are with the EUROfusion Consortium, PPPT, Boltzmannstr. 2, D-85748 Garching, Germany and the Max-PlanckInstitut für Plasmaphysik, Boltzmannstr. 2, D-85748 Garching, Germany (e-mail thomas.franke@euro-fusion.org).

Ch. Bachmann, and G. Federici are with the EUROfusion Consortium, PPPT, Boltzmannstr. 2, D-85748 Garching, Germany.

P. Agostinetti, P. Sonato and P. Vincenzi are with the Consorzio RFX (CNR, ENEA, INFN, Università di Padova, Acciaierie Venete SpA), Corso Stati Uniti 4 - 35127 Padova, Italy.

G. Aiello, G. Grossetti, T. Scherer and D. Strauss are with the IAMAWP, Karlsruhe Institute of Technology (KIT), Kaiserstr. 12, 76131 Karlsruhe, Germany.

K. Avramidis and J. Jelonnek are with the IHM, Karlsruhe Institute of Technology (KIT), Kaiserstr. 12, 76131 Karlsruhe, Germany

A. Bruschi, S. Garavaglia and G. Granucci are with the Institute of Plasma Physics "P. Caldirola”, National Research Council of Italy, 20162 Milan, Italy.

J.-M. Noterdaeme is with the Max-Planck-Institut für Plasmaphysik, Boltzmannstr. 2, D-85748 Garching, Germany and Department of Applied Physics, University Ghent, 9052 Ghent, Belgium.

A. Simonin is with the CEA-IRFM, F-13108 Saint-Paul-Lez-Durance, France.

M. Q. Tran is with the Swiss Plasma Center (SPC), EPFL, CH-1015 Lausanne, Switzerland.

A. Valentine and S. Zheng are with the Culham Centre for Fusion Energy, Culham Science Centre, Abingdon, Oxfordshire, OX14 3DB, United Kingdom. configurations and design variants will have an impact on the performances for the whole fusion plant. It shall be noted that the basis for the H\&CD integrated design and system development is the actual version of the European fusion electricity roadmap [5].

The project also elaborates on H\&CD efficiency improvements which will reduce the recirculating power fraction in future fusion power plants. Different studies under investigation will be discussed such as, for NBI the photoneutralization and, for EC novel concepts for gyrotron multistage depressed collector.

Index Terms - Fusion Power Plant, DEMO, Heating \& Current Drive, Electron Cyclotron, Neutral Beam Injection, Ion Cyclotron, wall-plug efficiencies

\section{INTRODUCTION}

TN the course of the pre-conceptual design of the 1 DEMOnstration fusion power plant several new and innovative DEMO H\&CD systems and subsystem designs are under development. In this paper the impact of their configurations to the DEMO plant is described for some of the recently studied aspects. Evidence for this is the wallplug efficiency as part of the technical specification of the H\&CD systems. The efficiency will have a major impact on the internal recirculating power of the DEMO balance of plant [6]. Assuming that the net electric power which DEMO is aiming to deliver to the grid by 2050 is around 300 to $500 \mathrm{MW}$ a higher wall-plug efficiency can help to reduce the fusion power to be generated. Thus would enable slightly decrease the major radius of the machine, provided that it is compatible with divertor limits [7]. Therefore one major goal of DEMO H\&CD system developments is to aim for high efficiency.

DEMO (baseline 2017) is a machine of $8.9 \mathrm{~m}$ major and 2.9 $\mathrm{m}$ minor radius, for a pulsed operation with $7200 \mathrm{~s}$ burn time and $<600 \mathrm{~s}$ dwell time, with a toroidal field of $4.9 \mathrm{~T}$, a target H\&CD system efficiency (wall plug efficiency) of 40 $\%$ and a plasma current of 19 MA. Further details on the key EU DEMO design is reported in [1].

In the frame of the Power Plant Physics and Technology (PPPT) for the H\&CD Research \& Development (R\&D) work and their (Pre-) Conceptual Designs [8] a number of studies were conducted to elaborate the applicability of innovative systems based on the experience gathered so far from ITER conceptual design and R\&D. Some of the most 
relevant recent design assessments for the DEMO H\&CD are described in this paper. Since the studies are comprehensive and done by several groups the results are updated and exchanged among the teams on a regular basis. In the following chapters a first overview of these studies will be summarized, and more details are given in the various references attached to each paragraph.

\section{DEMO AdvanCED NEUTRAL BEAM (NB) CONFIGURATION TO PERFORMANCE STUDIES}

For the DEMO NBI system two approaches are considered: a conventional NB system with beam neutralization on gas target (ITER-like system) [9],[10],[11], and a more challenging NB system based on beam photoneutralization [12],[13] which if feasible would offer a huge benefit of much higher neutralization efficiency compared to state-of-the-art technology, i.e. gas neutralizers. Knowing the related risks and challenges for this development and the tight timeline and development costs for mock-ups it was decided to invest in such innovative concept because it also offers great advantages. The requirements to be satisfied are quite different especially for the optical parts, which are new for an NB injector as e.g. optical tables, high precision optical cavities [14],[15] of high finesse with highly reflective mirrors and extremely high power lasers, which are at the leading edge of technology. The attempt was started with a development to integrate such a system and at reduced scale to highlight the different issues and estimate the concept feasibility.

In order to integrate such system into a fusion power plant detailed studies for the most important issues are on-going, these are in particular (i) port integration studies especially focusing on the required breeding blanket system openings, (ii) the heat load estimates on components like NB liners and the plasma facing components within the duct, (iii) the assessment of duct pump options to reduce the re-ionization losses, (iv) the finding of feasible remote handling schemes and (v) the conduction of neutronics studies, including the shut-down dose rates to estimate the amount of radiation after plasma operations, thus achieving by proper material selection and sufficient shielding.

\section{A. NBI conceptual design}

The NB systems were compared below (Table I) for two options, an ITER-like NB [11], an advanced DEMO-NB [12],[13] and a model used in previous simulations called 'METIS Ref.'(see [16],[17]). The ITER-like NB injector is characterized by a high extracted current density and a high beam voltage, but both were found to be contradictory for a highly reliable power plant operation over a long operation period. Also the use of only one NB source has possible drawbacks in terms of reliability and it is more difficult to construct in terms of high thermal loads on the grids and their thermal expansion. The fact that if one or even more of their Radio Frequency (RF) driver fails the whole injector is out of operation lead to an alternative modular approach for DEMO. Therefore, the DEMO advanced concept has several RF sources, and if one of the drivers would fail, the whole injector would be still operable losing only a limited amount of the nominal injected power. Also a new concept of RF ion source is under study with Helicon plasma drivers, and the first results are encouraging [18],[19].

The extracted current density of the DEMO advanced injector was balanced to a lower value in order to have the possibility to reduce the filling gas pressure in the ion source, with a consequent reduction of beam stripping losses through the accelerator. Moreover, the beam energy was decreased by $20 \%$ to improve the reliability of the whole system regarding voltage holding, as this aspect was identified as one of the most critical issues. Because of higher efficiencies one of the DEMO advanced injectors is still able to produce sufficient amount of power so that with the same number of injectors, i.e. three, the total injected power of $50 \mathrm{MW}$ can be achieved. This thanks also to the application of laser-neutralizers presently under development, whereby first proof-of-principle experiments show promising results.

TABLE I

NB INJECTOR OPTIONS (MAIN PARAMETERS)

\begin{tabular}{l|l|l|l}
\hline \hline NB injector parameter & ITER-like & $\begin{array}{l}\text { DEMO } \\
\text { advanced }\end{array}$ & $\begin{array}{l}\text { METIS } \\
\text { Ref.* }\end{array}$ \\
\hline $\begin{array}{l}\text { Extracted D Current Density } \\
{\left[\mathrm{A} / \mathrm{m}^{2}\right]}\end{array}$ & 286 & 200 & - \\
Number of sub-sources & 1 & 20 & 1 \\
Tangency radius [mm] & $7090\left(30^{\circ}\right)$ & $7090\left(30^{\circ}\right)$ & 8000 \\
Beam voltage [kV] & 1000 & 800 & 1000 \\
Power per injector [MW] & 16.5 & 16.8 & 25 \\
Number of injectors & 3 & 3 & 2 \\
Neutralization efficiency [\%] & 55 & 70 & - \\
Injector wall-plug efficiency [\%] & 26 & 51 & - \\
& 49.5 & 50.4 & 50.0 \\
Total Injected power from NB & & \\
system [MW] & & \\
\hline \hline
\end{tabular}

* "METIS Ref." is the beam used in all the work done so far within WPPMI (a work group under PPPT) with METIS system code. It has been set to fit the desired scenario computed by PROCESS code (a DEMO reactor simulation code), but it does not correspond to any NB system design.

The arrangement of the NB injectors is shown in Fig. 1.

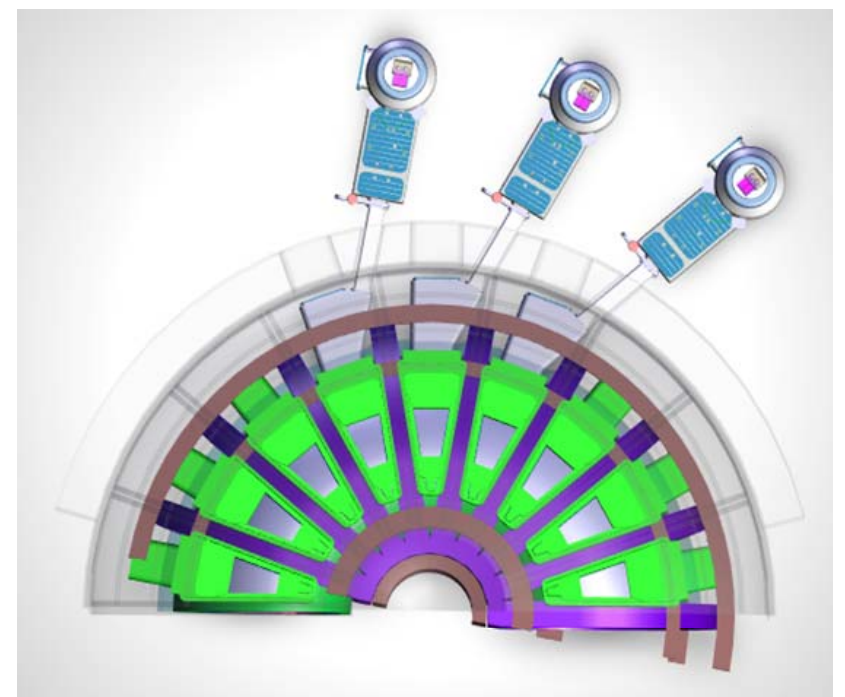

Fig. 1. EU DEMO1 2015 with 3 NB injectors as viewed from the top

In contrast to ITER, it is foreseen to have an inclined part of the NB port for the liner and dedicated port duct pumps and a bigger radial part for maintenance access.

\section{B. NBI physics simulations}

Based on the parameters given by the PPPT for the DEMO1 pulsed scenario such as temperature and density 
profiles [see Fig. 2, (left)] the beam-plasma interaction was studied with the fast tokamak simulator METIS [17]. For this purpose only the plasma alpha particle heating for the burning plasma (with a fusion gain factor of $Q=40$ ) and NBI heating was considered, whereas the EC heating was set to zero. [see Fig. 2, (right)].
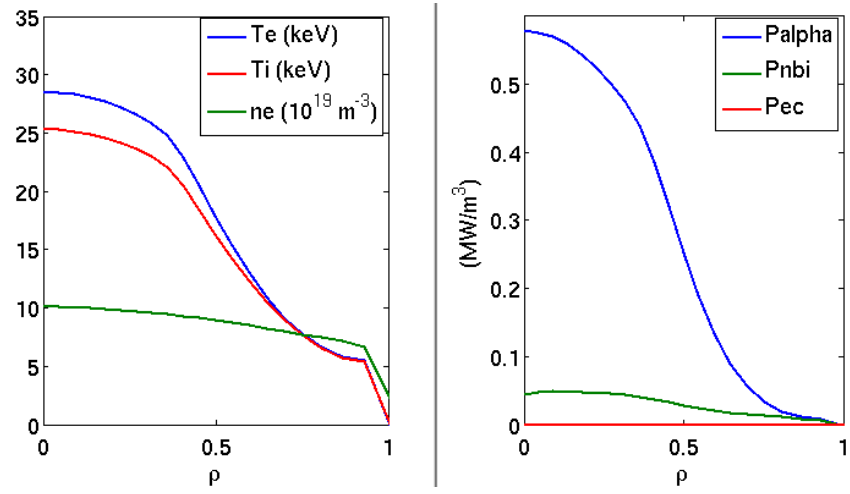

Fig. 2. NB simulations in METIS, plots for Ti, Te, ne (left) and Palpha, Pnbi, Pec (right)

The simulations were compared for different parameters, see Fig. 3, in which the NBI power (pnbi) for all three studied system options were kept at $50 \mathrm{MW}$. The fraction of absorbed power in the plasma is always $\sim 100 \%$ since losses due to fast ions are negligible. The fusion power (pfus_nbi) coming from NBI-plasma interaction is only slightly affected and this results from the beam trajectory (xnbi) differences. The overall plasma is not influenced much by these changes, although the NB power deposition, induced current, etc. are different. The NB rotation part (snbi) in Fig. 3 is given not in absolute but only relative values. At the moment, since the 'natural' rotation of the plasma is not known, the impact of the NB rotation cannot be estimated and will be part of future studies. The driven current by auxiliary HCD NBI power is higher for the high energy beams $(1 \mathrm{MeV})$ compared to the DEMO advanced (800 $\mathrm{keV}$ ) version. For a pulsed DEMO machine this is of minor importance, whereas for a steady state machine indeed, it would have a huge impact on the machine design and performance.
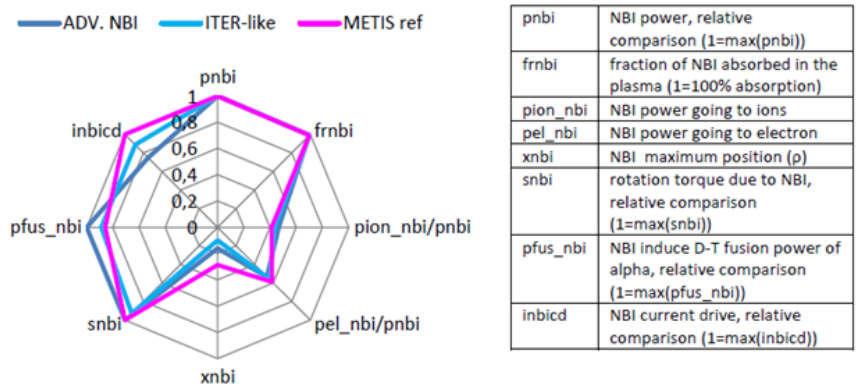

Fig. 3. Radar chart of the ITER-like NB vs. DEMO advanced concept

The values gained in Fig. 3 with METIS calculations were later refined with other (BBNBI and ASCOT) codes.

With this physics based comparison it was concluded that the advanced NBI does not show major deviations compared to the ITER-like solution and the further implementation to DEMO is favorable and recommendable [16].

In the following study the integration of the beam was performed with different (in total 3) options [12] of which two are described in Fig. 4 below. The two shown options are different in injection angle. The comparison was required in order to see the drawbacks of the one or other solution.
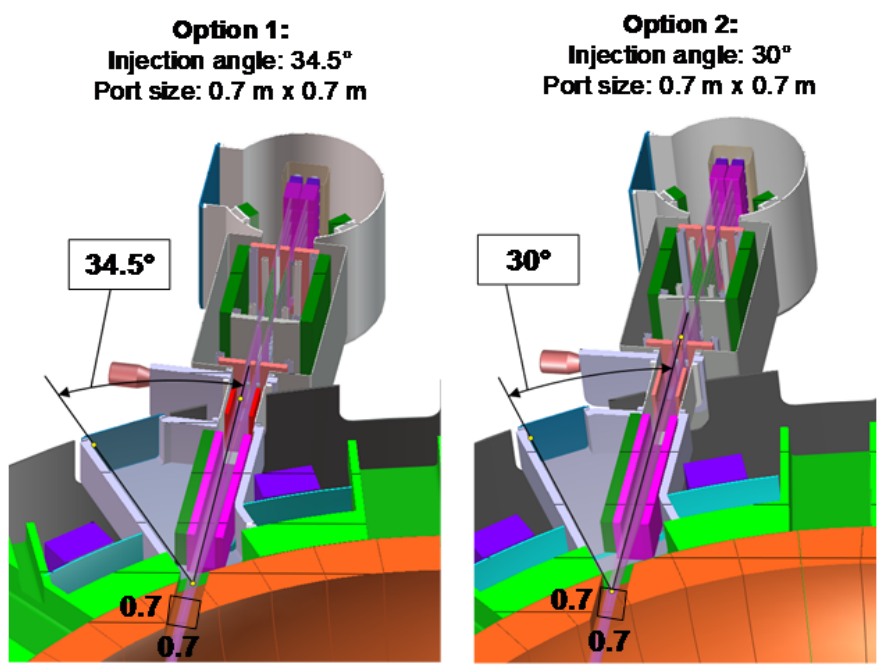

Fig. 4. Options for injection angles and integration studies shown without bioshield (opt. 2 was selected for further studies)

A physics comparison was done with METIS for both options as shown in Fig. 4 and the result can be seen in Fig. 5. It shows that independently of the tangential angle $\left(30^{\circ}\right.$ vs. $34.5^{\circ}$ ) the NBI performance is similar, except for the reactions of the fast ions with the plasma (which represents only $1 \%$ of the total fusion power and is therefore negligible) and for the current drive, which for a pulsed DEMO is - as explained before - of minor interest.
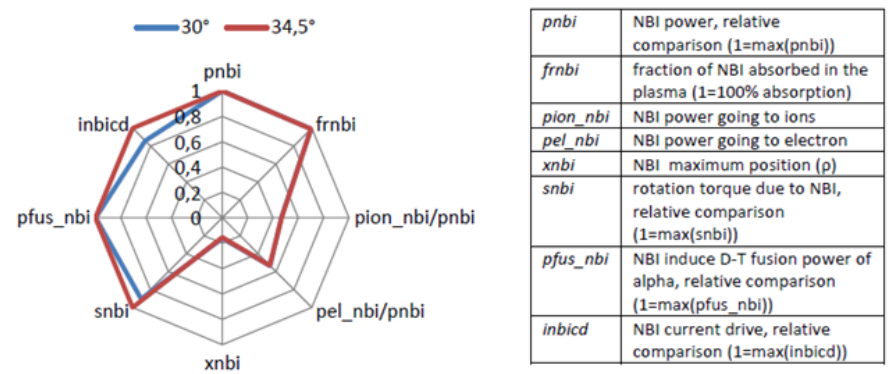

Fig. 5. Radar chart of the NB Option 1 compared to the Option 2

Finally Fig. 6 shows a viewgraph for the studies of the shine-through losses to the opposite wall (through the plasma), which were found for both options as negligible. The simulations done with the codes BBNBI and ASCOT are explained in [16].
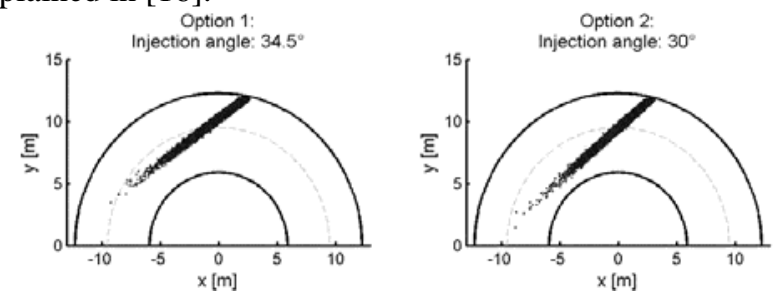

Fig. 6. Shine-through studies of the 2 NB options

For the ramp-up phase presently studies are on-going to find the right timing at which the NBI can be switched on during the ramp-up phase to avoid too high shine-through losses. This is very likely only at the end of the plasma ramp-up phase possible for the NB with $800 \mathrm{keV}$ beam 
energy. Indeed a lower NB energy, the injection trajectory which does not intersect the inner wall and the large DEMO volume would permit an earlier switch on of the beam. If this combined low and high beam energy scenario is not applicable for DEMO this consequently requires another H\&CD system (like EC) to form a dense enough plasma which would then allow the high energy beam NBI to inject power to assist the L-H transition heating.

\section{NBI neutronics studies}

For the two NBI options as shown before Monte-Carlo NParticle (MCNP) studies were also performed to allow the estimation of the neutron fluxes and fluences, the displacements per atom (dpa) in the materials and to assess the material lifetimes. Furthermore the shut-down dose rate after operation was computed. The results for the neutron streaming analysis are shown in Fig. 7.

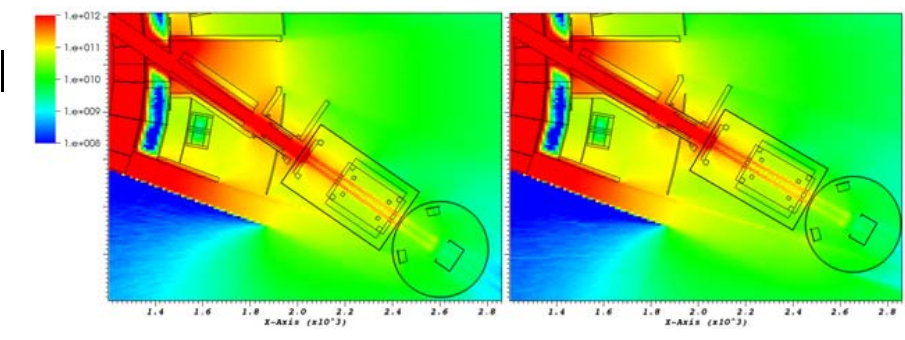

Fig. 7. Neutron streaming analysis NBI option 1 (left) \& 2 (right side). Neutron flux $\left[\mathrm{n} /\left(\mathrm{cm}^{2} \cdot \mathrm{s}\right)\right]: 1 \cdot 10^{8}$ (blue) to $1 \cdot 10^{12}$ (red), everything below $10^{8}$ and above, up to a maximum of $10^{15}$, was cut off in the photograph. Each $\mathrm{x}$-axis represents the distance from the machine center in the range from $13.0 \mathrm{~m}$ to $28.3 \mathrm{~m}$

The immediate conclusion from the two photographs is that both injector options behave similar, and to see the difference better, a superposition of both results was made and the differences are seen then in Fig. 8. These MCNP studies led to two other results, not shown here. The results show that the liner materials dpa's are within the limits given for the lifetime of this material in DEMO. In addition the Toroidal Field (TF) coil heating slightly exceeds the limits so that for some parts of the NB port additional shielding needs to be added. The studies are on-going.

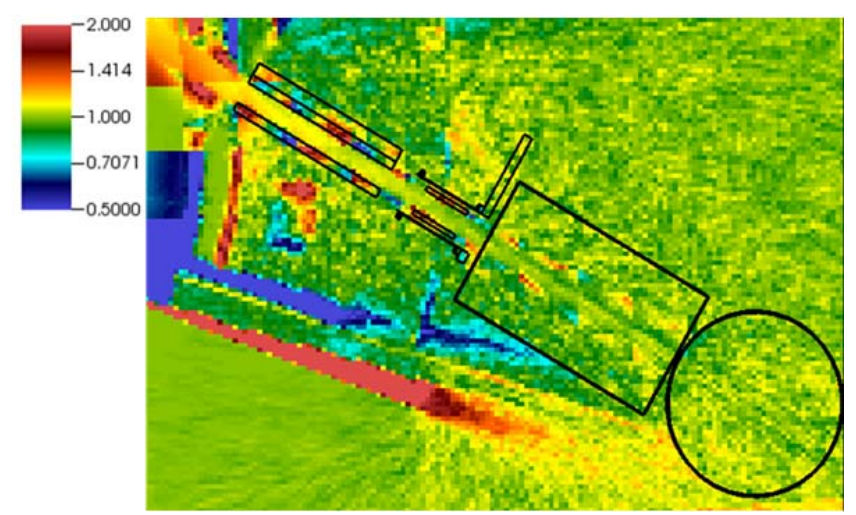

Fig. 8. Neutron streaming comparison option 2 / option 1

Legend: range from 0.5 (dark blue) to 2.0 (violet red) is the ratio of neutron flux of option 2 to option 1

\section{ELECTRON CYCLOTRON (EC) CONFIGURATIONS FOR DEFINITION OF DEMO (PLANT) LAYOUT}

Taking into account the lessons learned from ITER EC [20] a similar approach as for NB was done for the EC system. A number of physics studies (not discussed here) were conducted together with conceptual layout studies [21],[22]. Also different options for the configurations were investigated [23]. The EC physics functions to be fulfilled for DEMO and investigated so far are on the EC plasma breakdown, EC current drive, L-H heating, ramp-up and down assistance, neo-classical tearing mode (NTM) and sawtooth (ST) control and control of radiative instabilities.

\section{A. EC plant layout}

Two different EC plant layouts were studied. The first EC plant layout proposal is to locate three gyrotron (RF) buildings at $120^{\circ}$ around the tokamak and the second plant layout in which the EC power sources are allocated in only one building in the south of the tokamak according is shown in draft plant layout, see Fig. 9. In both cases a minimum distance between tokamak and gyrotron building(s) has to be considered as described below. For the connections from the EC power sources (gyrotrons) to the EC launchers in DEMO; two solutions are under investigation: evacuated waveguides (EWGs) as in ITER and Quasi-optical (QO) Transmission Lines (TLs) (as in Wendelstein 7-X, Greifswald, Germany). In contrast to W7-X, where dry air is the transmission medium, in DEMO the EC beams should be transmitted in vacuum for Tritium safety considerations and because of higher power densities. A preliminary conceptual design of the Evacuated Quasi-optical (EQO) TL based on mirror (Fig. 10) confocal layout can be found in [21]. The QO Multi-Beam TL, able to arrange up to 8 beams in the present design, reduces the number objects to align with respect to EGWs and easing the task to cope with problems due the thermal effects.

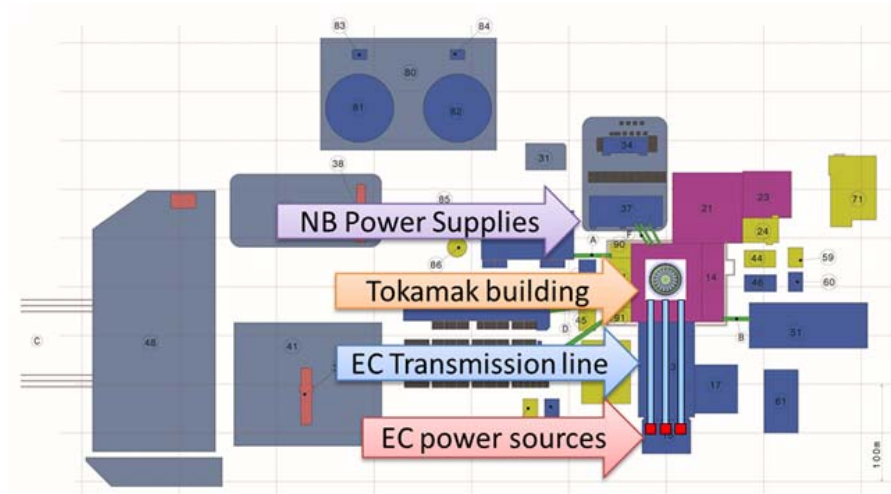

Fig. 9. Provisional DEMO plant layout with EC and NB systems (other EC configurations were also studied, as described in the text before)

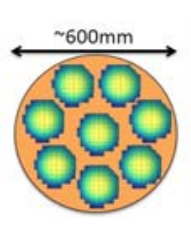

\begin{abstract}
Fig. 10. Sketch of the minimum envelope circular surface of the Evacuated Quasi-optical (EQO) Transmission Line (TL) to host 8 individual beams (total $\sim 600 \mathrm{~mm}$ diameter, tbc.: $+25 \%$ in diameter to reduce losses)
\end{abstract}

The minimum length of the TL is depending also on the stray magnetic field of the tokamak as the EC gyrotrons operation is limited by the stray magnetic field. In order to find a position for the gyrotrons compatible with their operations a magnetic field map was computed (see Fig. 11).

- Gyrotron requirements (G = Gauss) [24]-[26]:

$\mathrm{B}_{\mathrm{R}}<2 \mathrm{G}$ (radial), $\mathrm{B}_{\mathrm{Z}}<5 \mathrm{G}$ (axial), up to $10 \mathrm{G}$ (still 
acceptable, to be confirmed)

- Torus-Gyrotron distance as simulated (see Fig. 11)

DEMO $>120 \mathrm{~m}$ (for comparison in ITER is $105-135$ $\mathrm{m})$

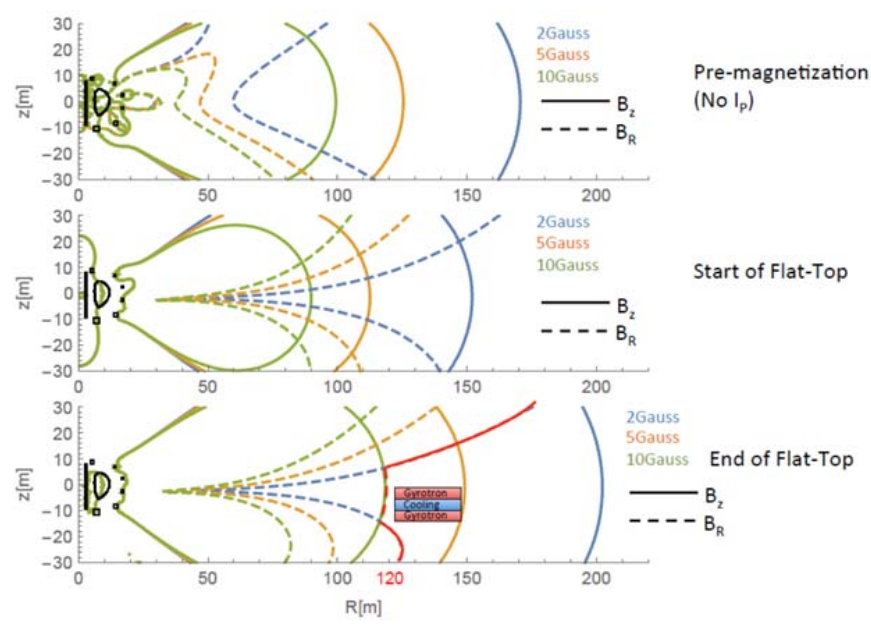

Fig. 11. DEMO stray magnetic field map for radial and axial field

\section{B. EC launcher configurations}

Two types (out of some others) of possible DEMO EC port plug configurations were studied in more detail, both which stop behind the blanket and for which the blanket has funnel type openings to cope with the beam stearing of about $+/-15^{\circ}$ (see Fig. 12).

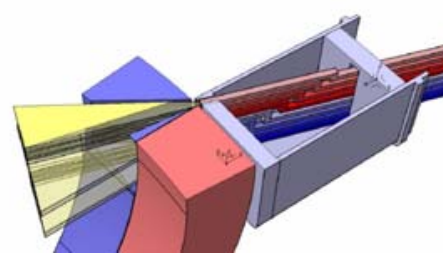

Configuration A: a stack of 8 antennas

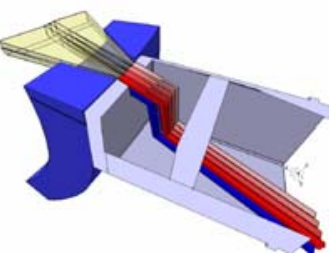

Configuration B: a stack of 2 rows of 4 antennas
Fig. 12. EC equatorial port plug options (isometric view)

MCNP calculations were performed for both port plug options. First results show that the neutron shielding is not sufficient especially for the configuration $A$ and further improvements are foreseen before the study will be repeated for an improved design. Solutions are envisaged to be feasible by dog-leg structures and including more neutron shield blocks in empty spaces. A different configuration with the aim to reduce the impact due to neutron streaming and foreseeing an integrated blanket design of the port plug with the blanket system will also be considered. Different design options are foreseen for the antennas: one is based on simple truncated waveguides (TW) at fixed orientation exploiting the multi-frequency and/or step-tunable gyrotrons for changing the deposition location in the plasma (in the last case requiring Brewster-angle RF windows, see Section E). The TW length is free of constraints, it can be used to cover a frequency range of several $\mathrm{GHz}$ and no movable mirrors are foreseen.

The other is based on the use of Remote Steering Antennas (RSA), designed for a single frequency.

\section{EC remote steering antenna}

The big advantage of the remote-steering concept in
DEMO compared to a front-steering mechanism as in ITER is that no movable plasma facing parts exist. The following characteristic points need to be considered for the DEMO design:

- In-Vessel Waveguides (WGs) implemented in equatorial port plugs with dog-leg structures.

- In-Vessel WGs at the back-end connected to the Remote Steering Antenna (RSA) mechanisms.

- RSA antennae connected to Evacuated Quasi-optical Transmission Lines (EQO TLs) in underground duct up to the RF building.

- Neutron shield blocks can be inserted around the WGs to protect overheating in Toroidal Field (TF) coils and vacuum vessel (VV).

The following specific points are to be considered for the RSA (pre-) conceptual design studies:

- Different types of RSA are required if different frequencies of the gyrotrons are chosen for different tasks.

- The optimized length is depending on frequency and cross-section of the RSA and important in order to place the RSA optical box outside the bioshield (see Fig. 13).

- The RSA are connected to diamond windows (Tritium and vacuum barrier) installed between RSA optical boxes and EC EQO TLs.

- RSA and diamond windows shall be well accessible in the EC port cell outside the bioshield.

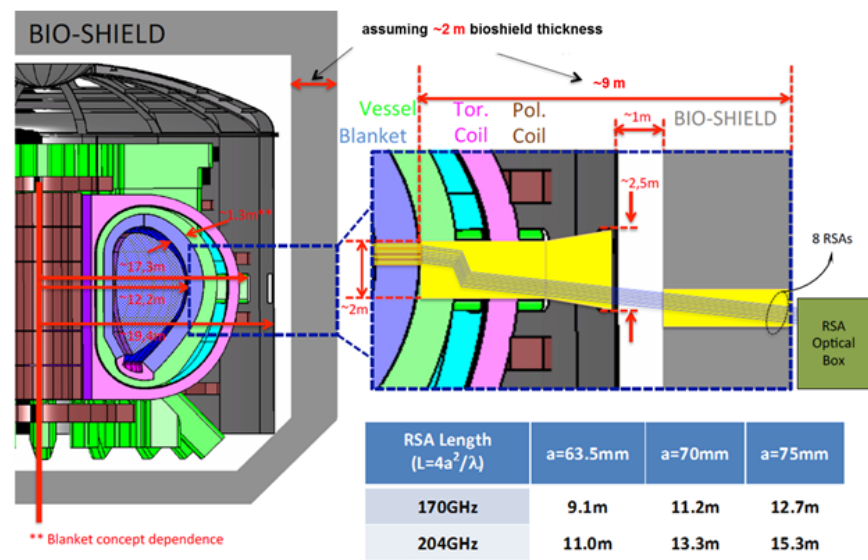

Fig. 13. Remote Steering Antenna (RSA) design options

Table in Fig. 13 shows the lengths of the RSA for different waveguide sizes ' $a$ ' and for two possible DEMO gyrotron frequencies $170 \mathrm{GHz}$ and $204 \mathrm{GHz}$. According to the calculated antenna lengths the integration of all waveguide options as shown can be approved to be feasible.

\section{EC gyrotron coaxial-cavity technology and multi stage depressed collector concept}

In future the coaxial-cavity gyrotron technology shall help to achieve multi-megawatt, multi-purpose and, at the same time, frequency step-tunable gyrotrons operating at an output power significantly above $1 \mathrm{MW}$ and an operating frequency up to $240 \mathrm{GHz}$ [27]. For example, new cooling technologies and advanced key components, such as a Magnetron Injection Gun (MIG) free of trapped electrons 
[28] with advanced type of emitter ring [29] and, in parallel, an innovative inverse MIG [30], are under development to achieve that goal.

To recover the kinetic energy from the spent electron beam and, hence, to increase the total efficiency of gyrotrons, the single stage depressed collector (SDC) design approach is so far the only applicable solution. It is implemented in all recent gyrotrons, e.g. for Wendelstein 7$\mathrm{X}$, Greifswald, Germany and it will be implemented for ITER gyrotrons. Today's efficiency of fusion gyrotrons is around $50 \%$. The target for DEMO is a total efficiency of the gyrotrons of better than $60 \%$. It is possible to achieve by the development of multi-stage depressed collector concepts.

A novel solution is based on the $\boldsymbol{E} \times \boldsymbol{B}$ drift concept [31], in which an effective energy sorting of the electrons becomes feasible and the secondary electrons can be handled. Various design ideas were discussed and a two stage design looks most promising (see Fig. 14) [32]-[34].

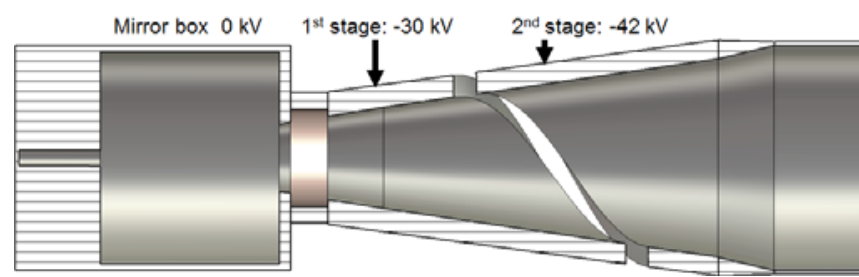

Fig. 14. Two-Stage Gyrotron Collector based on E×B

The achievable collector efficiency of this innovative concept might exceed $75 \%$ and the resulting total gyrotron efficiencies based on this concept might reach $>60 \%$. It should also be noted that the designs under study are compatible with the levels of the stray magnetic field defined in section III-A and that the required magnetic field for the function of the $\boldsymbol{E} \times \boldsymbol{B}$ concept is small and not expected to affect the neighboring gyrotrons [35]. Further studies are required and a proof-of-principle is in the long term planned to confirm the validity of the concept.

\section{E. EC diamond windows}

The conventional single chemical vapor deposition (CVD) diamond disk consists of a disk, perpendicular to the axis of the waveguide where the beam propagates, having a thickness resonant with the beam wavelength. The thickness of the disk must be an integer multiple of half the wavelength of the beam inside the material. This configuration is used for the fixed frequency operation in ITER where WGs with $50 \mathrm{~mm} / 63.5 \mathrm{~mm}$ inner diameter are foreseen. A $63.5 \mathrm{~mm}$ WG requires a disc with a diameter of $80 \mathrm{~mm}$.

The conventional configuration of the disk is one option for DEMO compatible with the launchers based on the RSA concept and used together with multi-frequency gyrotrons. In the latter context, the resonance condition must be respected with all the beam wavelengths generated by the gyrotrons.

The second option of disk configuration for DEMO is the Brewster-angle diamond disc. In the case of diamond, the Brewster angle, i.e. the angle between the normal to the disc and the axis of the WGs, is $67.2^{\circ}$. This disk configuration is required with frequency step tunable gyrotrons able to switch their operating frequency in steps of around 2-3 GHz.

Key challenges in the Brewster-angle window development towards long pulse operation are the manufacturing of sufficiently large diamond discs, the proper joining of such discs to the WGs and the design of a cooling layout able to guarantee a proper removal of the heat absorbed during beam transmission. The manufacturing of large disks is challenging. As the target is a diamond disc suited for a $63.5 \mathrm{~mm}$ inner diameter WG at the Brewster angle, a disc of $180 \mathrm{~mm}$ would be required. In collaboration with industrial partners, different manufacturing options are currently under investigation.

The cooling performance of Brewster-angle windows was first studied for the $50 \mathrm{~mm}$ inner diameter WGs [36]. As an example, the temperature distribution corresponding to the case of elliptical cooling channels is reported in Fig. 15. The main conclusion of the study was that cooling channels that follow the skewed position of the disc are required; otherwise the temperatures in the diamond disc due to the mm-wave losses result in values beyond the limit of 250 $300{ }^{\circ} \mathrm{C}$ for diamond, at which the dielectric properties of diamond start degrading.

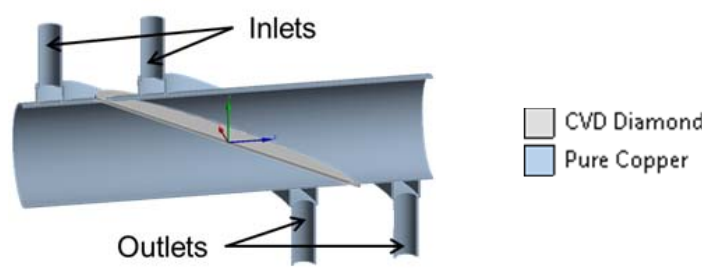

$\left[{ }^{\circ} \mathrm{C}\right]$

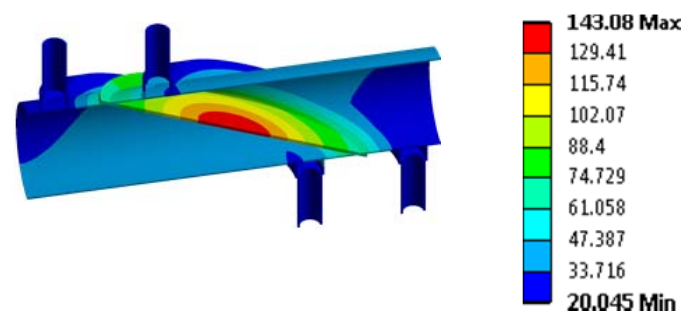

Fig. 15. Power performance of large synthetic diamond windows (Example of FEM analysis result)

\section{ION CYCLOTRON (IC) DISTRIBUTED ANTENNA}

The toroidally distributed IC antenna is a new concept for DEMO and described e.g. in [8] and [37]. Compared to the conventional approach used in present day machines or foreseen in ITER (where the antenna occupies equatorial ports), the concept has many physics advantages: it allows for an improved coupling by being able to work with low $\mathrm{k}_{/ / \text {, }}$ and strongly reduces the occurrence of sheaths and impurity production. Simulations indicate that to couple about 40-60 MW, the voltage on the straps is of the order of $15 \mathrm{kV}$ [37]. From an integration point of view, the distributed antenna offers the following advantages: minimum impact on the blanket function, with the advantages regarding neutron shielding and tritium breeding ratio, modularity, matching the blanket segmentation (to facilitate Remote Maintenance), and no need of using ports in the vessel (which are limited and in high demand). Several options are under investigation and the antenna design is presently 
under studies [38]. Further investigations are being undertaken and can only be discussed at a later stage of the project after consolidation and further studies on physics, integration, toroidal layout of the antenna sectors, antenna strap and Faraday Shield (FS) design and the corresponding layout of the Transmission Lines (TLs). With the different design options Tritium breeding ratio (TBR) calculations, already carried out for the antenna only, shall be refined in due time to take into account the impact of the different TLs feeding schemes. Available remote handling schemes and the TL and antenna integration in the breeding blanket are another prerequisite and therefore more in depth integration studies have to be carried out.

\section{CONCLUSION}

Novel and innovative designs of H\&CD systems and subsystems and their impact on other systems and interfaces were discussed. Studies analyzed plasma interaction for IC, EC and NB. Stray magnetic fields for the EC and neutron calculations for NB and EC systems were performed and suggestions for further work were formulated.

Depending on the results and progress of the manufacturing of large Brewster windows test samples, the implementation of frequency step tunable gyrotrons might be applicable to DEMO. The focus was given to efficiency improvements in NB and EC systems. Pathways to it by NB photo-neutralization or $\boldsymbol{E} \times \boldsymbol{B}$ staged depressed collector designs for EC were shown and first results are available.

The scoping studies were undertaken on most promising design options after discussion in the design teams of H\&CD work package but also by interdisciplinary work with the teams of the other PPPT work packages.

\section{ACKNOWLEDGEMENT}

This work has been carried out within the framework of the EUROfusion Consortium and has received funding from the Euratom research and training programme 2014-2018 under grant agreement No 633053. The reviews and opinions expressed herein do not necessarily reflect those of the European Commission. The authors thank the contributors of their teams and the colleagues of the other PPPT workpackages and the Project Management Unit (PMU) of EUROfusion for their strong support and fruitful cooperation.

\section{REFERENCES}

[1] G. Federici, et al., 'Overview of EU DEMO design and R\&D activities', Fus. Eng. Design, vol. 89, pp. 882-889, 2014, https://doi.org/10.1016/j.fusengdes.2014.01.070

[2] A.J.H. Donné et al., Scientific and technical challenges on the road towards fusion electricity, Journal of Instrumentation, Volume 12, October 2017, http://iopscience.iop.org/article/10.1088/1748$\underline{0221 / 12 / 10 / C 10008}$

[3] R. Wenninger, et al., 'The physics and technology basis entering European system code studies for DEMO', Nucl. Fusion 57, 016011, 2017.

[4] P. Vincenzi, et al, 'EU DEMO transient phases: main constraints and heating mix studies for ramp-up and ramp-down`, 2017, Fus. Eng. Design., in press, https://doi.org/10.1016/j.fusengdes.2017.02.064

| [5] F. Romanelli, et al., 'Fusion Electricity - A roadmap to the realisation of fusion energy, European Fusion Development Agreement (EFDA) 2012, ISBN 978-3-00-040720-8.

[6] M. Kovari, R. Kemp, H. Lux, P. Knight, J. Morris, D.J. Ward, '”PROCESS": A systems code for fusion power plants-Part 1:
Physics'. Fusion Eng Des. 2014 Dec ; 89(12):3054-69. http://www.sciencedirect.com/science/article/pii/S0920379614005961

[7] T. Franke, et al., 'On the Present Status of the EU DEMO H\&CD Systems, Technology, Functions and Mix', 26th SOFE conference, Austin, Texas USA (2015), https://doi.org/10.1109/SOFE.2015.7482410

[8] M. Q. Tran, et al., 'EU DEMO Heating and Current Drive', Physics and Technology, Conf. Proc. 26th IAEA Fusion Energy Conference, October 17th - 22nd, 2016 Kyoto, Japan.

http://www.euro-fusionscipub.org/archives/eurofusion/eu-demoheating-and-current-drive-physics-and-technology

[9] R. S. Hemsworth et al., 'Overview of the design of the ITER heating neutral beam injectors', New Journal of Physics, vol. 19, pp. 2017. http://iopscience.iop.org/article/10.1088/1367-2630/19/2/025005

[10] M. J. Singh et al., 'Heating neutral beams for ITER: negative ion sources to tune fusion plasmas', New Journal of Physics, vol. 19, pp. 2017. http://iopscience.iop.org/article/10.1088/1367-2630/aa639d

[11] U. Fantz, et al., 'Towards Powerful Negative Ion Beams at the Test Facility ELISE for the ITER and DEMO NBI Systems', Nucl. Fusion 57,116007

[12] P. Sonato, P. Agostinetti, T. Bolzonella, F. Cismondi, U. Fantz, A. Fassina, T. Franke, I. Furno, C. Hopf, I. Jenkins, Conceptual design of the DEMO neutral beam injectors: main developments and R\&D achievements, Nucl. Fusion 57 (2017) 056026

[13] P. Sonato, P. Agostinetti, U. Fantz, T. Franke, I. Furno, A. Simonin and M. Q. Tran, 'Conceptual design of the beam source for the DEMO Neutral Beam Injectors', New J. Phys. 18 (2016) 125002.

[14] A. Simonin, et al., 'Towards a new generation of neutral beam system for future fusion reactors', 27th IEEE Symposium On Fusion Engineering, 2017, Shanghai, China

[15] A. Simonin, et al., 'Negative ion source development for a photoneutralization based neutral beam system for future fusion reactors', New J. Phys. 18 (2016) 125005. https://doi.org/10.1088/1367-2630/18/12/125005

[16] P. Vincenzi et al., 'Comparison of Neutral Beam Injection options for EU DEMO pulsed scenario', P4.146, EPS Conf., 26-30 June 2017, Belfast, Ireland.

[17] J.F. Artaud, et al.,' The CRONOS suite of codes for integrated tokamak modelling', Nucl. Fusion 50 (2010) 043001.

[18] I. Furno, et al., 'Helicon wave-generated plasmas for negative ion beams for fusion', RFPPC 2017, Aix en Provence, France, submitted to EPJ woc.

[19] C. Marini, et al., 'Spectroscopic characterization of H2 and D2 helicon plasmas generated by a resonant antenna for neutral beam applications in fusion', Nucl. Fusion 57 (2017) 036024 (9pp), https://doi.org/10.1088/1741-4326/aa53eb

[20] T. Omori, M. A. Henderson, F. Albajar, S. Alberti, U. Baruah, T. S. Bigelow, et al. (2011), 'Overview of the ITER EC H\&CD system and its capabilities', Fusion Engineering and Design, 86(6-8), 951-954. doi:10.1016/j.fusengdes.2011.02.040.

[21] G. Granucci, et al.,' 'Conceptual Design of the EU DEMO EC-System: Main Developments and R\&D Achievements', accepted for publication on NF.

[22] G. Grossetti et al., 'DEMO Port Plug Design and Integration Studies', Conf. Proc. 26th IAEA Fusion Energy Conference, October 17th 22nd, 2016 Kyoto, Japan.

[23] S. Garavaglia, W. Bin, A. Bruschi, G. Granucci, G. Grossetti, J. Jelonnek, A. Moro, N. Rispoli, D. Strauss, Q. M. Tran and T. Franke, 'Preliminary Conceptual Design of DEMO EC System', AIP Conf. Proc. $1689 \quad 978-0-7354-1336-8 \quad$ (2015) 090009, https://doi.org/10.1063/1.4936546.

[24] S. Garavaglia et al., 'EU DEMO EC System Preliminary Conceptual Design', submitted to Fusion Eng. Des., special issue ISFNT 2017

[25] I. G. Pagonakis, F. Li, S. Illy, B. Piosczyk, S. Alberti, J.-P. Hogge, S. Kern, M. Henderson, C. Darbos, 'Study of the ITER stray magnetic field effect on the EU 170-GHz 2-MW coaxial cavity gyrotron', IEEE Trans. Plasma Sci., vol. 40, no. 7, pp. 1945-1956, Jul. 2012.

[26] B. Ell, I. Gr. Pagonakis, G. Gantenbein, S. Illy, M. Thumm, and J. Jelonnek, 'Study of the influence of stray magnetic fields on the operation of the European gyrotron for ITER', IEEE Transaction on Electron Devices, vol. 64, no. 8, pp. 3421-3428, Aug. 2017.

[27] J. Franck, K. A. Avramidis, I. Gr. Pagonakis, S. Illy, G. Gantenbein, M. Thumm, and J. Jelonnek, 'Multi-frequency design of a 2 MW coaxial-cavity gyrotron for DEMO', 40th Int. Conf. Infrared Millimeter THz Waves, 23-28 August 2015, Hong Kong, Conference proceedings 3132400 .

[28] I. Gr. Pagonakis, B. Piosczyk, J. Zhang, S. Illy, T. Rzesnicki, J.-P. Hogge, K. Avramidis, G. Gantenbein, M. Thumm, J. Jelonnek, 'Electron trapping mechanisms in magnetron injection guns', Physics of Plasmas 23, 23105, 2016; doi: 10.1063/1.4941705. 
[29] I. Gr. Pagonakis, S. Illy, M. Thumm, 'Influence of emitter ring manufacturing tolerances on electron beam quality of high power gyrotrons', Physics of Plasmas 23, 83103, 2016; doi: 10.1063/1.4959113

[30] J. Jelonnek, et al.,' Design considerations for future DEMO gyrotrons: A review on related gyrotron activities within EUROfusion', 29th Symposium on Fusion Technology (SOFT 2016), Praha, CZ, September 5-9, 2016.

[31] I. Gr. Pagonakis, et al., A New Concept for the Collection of an Electron Beam Configured by an Externally Applied Axial Magnetic Field, IEEE Trans. Plasma Science, vol. 36, no. 2, 2008.

[32] I. Gr. Pagonakis, et al., Multistage Depressed Collector Conceptual Design for Thin Magnetically Confined Electron Beams, Physics of Plasmas, vol. 23, 043114, 2016.

[33] C. Wu, I. G. Pagonakis, G. Gantenbein, S. Illy, M. Thumm, and J. Jelonnek, 'Conceptual designs of $\mathrm{E} \times \mathrm{B}$ multistage depressed collectors for gyrotrons', Physics of Plasmas, vol. 24, no. 4, p. 043102, 2017.

[34] C. Wu, I. G. Pagonakis, S. Illy, G. Gantenbein, M. Thumm, and J. Jelonnek, 'Novel multistage depressed collector for high power fusion gyrotrons based on an E×B drift concept', in 2017 IEEE International Vacuum Electronics Conference, April 2017, pp. 1-2.

[35] C. Wu, I. Gr. Pagonakis, K. A. Avramidis, G. Gantenbein, S. Illy, M. Thumm, J. Jelonnek, 'Gyrotron Multistage Depressed Collector Based on E $\times$ B Drift Concept using Azimuthal Electric Field --- Part I: Basic Design', Physics of Plasmas, under review.

[36] G. Aiello, et al., 'Cooling concepts for the CVD diamond Brewsterangle window', submitted to IRMMW-THz conference, Cancun, Mexico, 2017

[37] R. Ragona and A. Messiaen, 'Study of a distributed ICRF antenna system in DEMO', (2017) Proceedings of the 22nd RF Topical Conference, EPJ woc., https://www.epj-conferences.org/

[38] A. Bader, et al., 'Integrating a distributed antenna in DEMO: Requirements and challenges', Fusion Eng. Des. (2017), https://doi.org/10.1016/j.fusengdes.2017.03.035 